\title{
Adenocarcinoma of the minor salivary gland with concurrent MAML2 and EWSR1 alterations
}

\author{
Sangjoon Choi ${ }^{1}$, Junhun $\mathrm{Cho}^{1}$, Seung Eun Lee ${ }^{2}$, Chung-Hwan Baek ${ }^{3}$, Yi-Kyung Kim ${ }^{4}$, Hyung-Jin Kim ${ }^{4}$, Young Hyeh Ko ${ }^{1}$ \\ 1Department of Pathology and Translational Genomics, Samsung Medical Center, Sungkyunkwan University School of Medicine, Seoul; \\ 2Department of Pathology, Konkuk University Medical Center, Konkuk University School of Medicine, Seoul; \\ Departments of ${ }^{3}$ Otorhinolaryngology-Head and Neck Surgery and ${ }^{4}$ Radiology, Samsung Medical Center, Sungkyunkwan University School of Medicine, Seoul, Korea
}

\begin{abstract}
Salivary gland tumors are histologically diverse, and each entity has distinctive histopathological and molecular features. We report two cases of salivary gland tumors with unique histological and molecular findings, which have not been documented previously. The tumors were located in the base of the tongue in both patients. Most tumor cells were arranged in cords and nests, giving a trabecularlike appearance. Focally, glandular structures with intraluminal mucin and perivascular pseudorosette-like configurations were identified. Tumor cells had eosinophilic to clear cytoplasm, and showed mild nuclear atypia. They were positive for pancytokeratin and negative for S-100, p63, c-KIT, androgen receptor, and neuroendocrine markers. Multiple foci of capsular or lymphovascular invasion were identified, but the Ki-67 labeling index was low $(<5 \%)$. Fluorescence in situ hybridization revealed concurrent alterations of MAML2 and EWSR1 gene. Further investigations with a larger number of cases with similar histological and molecular features will accurately classify this tumor.
\end{abstract}

Key Words: Salivary gland; Neoplasm; EWSR1 gene; MAML2 gene

Received: June 1, 2020 Revised: December 1, 2020 Accepted: December 11, 2020

Corresponding Author: Young Hyeh Ko, MD, Department of Pathology and Translational Genomics, Samsung Medical Center, Sungkyunkwan University School of Medicine, 81 Irwon-ro, Gangnam-gu, Seoul 06351, Korea

Tel: +82-2-3410-2762, Fax: +82-2-3410-6396, E-mail: yhk0310@skku.edu

Salivary gland neoplasms are uncommon and account for only $3 \%-10 \%$ of head and neck tumors [1]. Tumors of the salivary gland are classified into distinct subgroups by their characteristic histological features, immunoprofiles, and genetic alterations, and the histological subgroups have unique clinical characteristics. In the current World Health Organization (WHO) classification of head and neck tumors, salivary gland tumors are classified into 21 malignant tumors, 11 benign tumors, and one tumor with uncertain malignant potential. However, tumors with characteristic histological types that do not meet the current diagnostic criteria are still being reported. Moreover, salivary gland neoplasms are often diagnostically challenging because of the morphologic overlap in some of the tumors and the presence of variants and mimickers. As immunostaining is usually not helpful in distinguishing peculiar cases, molecular analysis is necessary for accurate tumor classification.

We recently encountered two cases of minor salivary gland neoplasms with unique morphology, which do not meet the diagnostic criteria for a specific entity and are unclassifiable in the current WHO classification of head and neck tumors. The tumors were comprised of only epithelial components with trabecular arrangement and they formed focal mucin-producing glandular structures, and fluorescence in situ hybridization (FISH) analysis showed concurrent alteration of MAML2 gene as well as EWSR1 gene. This genetic change is exceedingly rare in head and neck tumors and only one case report has been published regarding dual gene rearrangement of MAML2 and EWSR1 [2].

Histological features of these tumors have not been reported previously, and their biological behaviors are unknown. Herein, we describe detailed clinicopathological and molecular characteristics of two minor salivary gland adenocarcinoma cases with MAML2 and EWSR1 alterations. We anticipate this report will extend the knowledge on this rare, but distinct tumor of the minor salivary gland. 


\section{CASE REPORT}

\section{Case 1}

A 48-year-old woman (patient 1 ) who had a history of hyperthyroidism and rheumatoid arthritis was referred to our hospital for examination of a mass in the base of her tongue, which was found accidentally during cervical magnetic resonance imaging (MRI) for neck pain evaluation. Clinically, the mass was palpable at the right sublingual gland site, but the patient had no symptoms.

MRI revealed a solid and cystic lesion measuring $3 \mathrm{~cm}$ in the right tongue base that seemed to have originated from the right sublingual gland. Positron emission tomography revealed ${ }^{18} \mathrm{~F}$ fluorodeoxyglucose uptake of the mass and mild hypermetabolic lymph nodes in the right level $1 \mathrm{~b}$ area, which were suspicious of metastasis. Distant metastasis to other organs was not identified. The patient subsequently underwent sublingual mass excision. She developed no local or distant recurrence of the disease 20 months after the surgery.

\section{Case 2}

Patient 2 was sent to us for consultation. This 63 -year-old otherwise healthy woman was admitted for chronic tonsillitis with pain.

Computed tomography revealed a submucosal mass measuring $2.1 \mathrm{~cm}$ in the right tongue base with focal cystic and necrotic areas. The bilateral neck lymph nodes were not significantly enlarged. Excisional biopsy was performed on the tongue base mass. The patient was referred to our hospital for further treatment. There was no evidence of recurrence or metastasis during the 14-month follow-up.

\section{Pathological findings}

We examined surgically resected specimens obtained from the right tongue base mass from each patient. The gross examination of the resected tumors showed relatively well-circumscribed, white tan-colored, solid masses with focal cystic changes and hemorrhage. Microscopically, the tumors were highly vascularized, and tumor cells were arranged in variably sized nests and cords that were separated by delicate fibrous septa containing thin blood vessels, which formed a trabecular-like pattern (Figs. 1A, 2A). Cytologically, the tumor cells were round to ovoid or sometimes spindle shaped and tended to have long and clear to abundant eosinophilic and granular cytoplasm. The tumor cells had small- to medium-sized nuclei with mild to moderate nuclear membrane irregularity, vesicular chromatin, and incon- spicuous nucleoli. The nuclei were aligned side by side perpendicular to the basement membrane around the vessels of the septum.

In the tumor from patient 1 , the nucleus was positioned away from the basement membrane and toward the fibrovascular septa, which imparted the appearance of reverse polarity. The blood vessels between tumor cell nests were usually thin, but perivascular hyalinization was prominent in some areas. Around these blood vessels, perivascular pseudorosette-like arrangements of nuclei were observed (Fig. 1B). Peripheral nuclear palisading was seen in this area. Focally, glandular structures with intraluminal and intracytoplasmic mucins were identified in both patients' tumors (Figs. 1C, 2B). There was no identifiable perineural invasion by the tumor cells; however, capsular invasion and lymphovascular invasion were occasionally found (Fig. 1D). Mitotic figures and tumor necrosis were not identified. There was no metastasis in the right level $1 \mathrm{~b}$ lymph node from patient 1.

The tumor cells showed diffuse positivity for cytokeratin (AE1/ AE3) (Figs. 1E, 2C). The pseudorosette-like components identified in patient 1 were weakly positive for cytokeratin. They also displayed CD99 immunoreactivity, which highlighted thick hyalinized blood vessels (Fig. 1F). Immunoshistochemical staining for chromogranin, CD56, smooth muscle actin (SMA), S-100, c-KIT, and androgen receptor (AR) were negative in tumor cells. The lack of p63 staining indicated an absence of myoepithelial differentiation in this tumor (Fig. 1G). CD34 staining revealed marked vascular proliferation between the tumor glands or nests. The Ki-67 labeling index was $<5 \%$ (Figs. 1H, 2D). Special staining for mucin using mucicarmine, periodic acid-Schiff, and periodic acid-Schiff-diastase showed the presence of intraluminal and cytoplasmic mucin in the tumor cells.

We performed break apart FISH analysis with MAML2, EWSR1, and ETV6 probes to confirm genetic changes of this tumor. A minimum of 50 tumor cell nuclei were evaluated. FISH results were interpreted as positive when $>10 \%$ of tumor cells showed a split of the red and green signals by $\geq 2$ signal diameters, or when a green signal deletion was identified. Both cases had evidence of MAML2 and EWSR1 alterations and were negative for ETV6. One case had alteration (partial deletion or unbalanced translocation) of MAML2 and translocation of EWSR1 (Fig. $3 \mathrm{~A}, \mathrm{~B})$. The other case showed translocations of MAML2 and EWSR1 (Fig. 3C, D).

Targeted next-generation sequencing (NGS) using CancerSCAN NGS panel was performed only for case 1 because of the lack of available tissue in case 2. Deep targeted sequencing detected 19 single nucleotide variants (SNV) including TOP1, PMS2, 

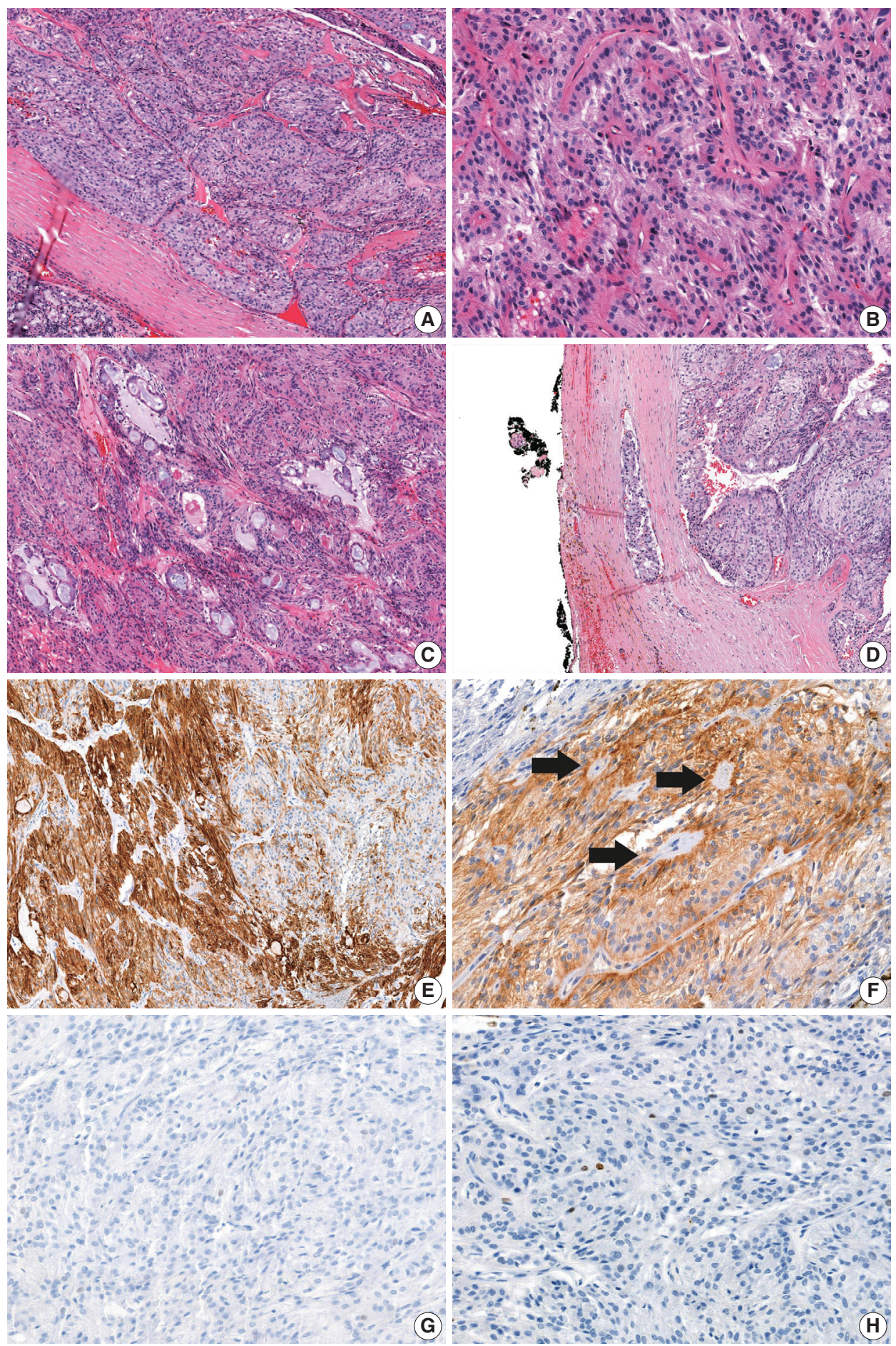

Fig. 1. Histopathological findings and immunostaining results for case 1. (A) Low-power image shows cords and nests of tumor cells separated by fibrous septa. The tumor cells appear round to polygonal and contain bland-looking nuclei with fine chromatin and abundant eosinophilic granular cytoplasm. Mucinous cells are scattered throughout the tumor. (B) In some areas, tumor cell nuclei show peripheral palisading around blood vessels, which give a perivascular pseudorosette-like appearance. (C) Focally, tumor cells show glandular differentiation and intraluminal mucin secretion. (D) Endolymphatic tumor emboli are found in the peritumoral fibrous capsule. (E) Cytokeratin (AE1/AE3) staining is positive in both the trabecular and glandular components (left side), but focal staining is observed in the rosettoid area (right side). (F) Tumor cells around the hyalinized vasculatures (arrows) with pseudorosette-like arrangement exhibit positive CD99 immunoreactivity. (G) Immunohistochemical staining for p63 reveals the lack of myoepithelial differentiation of this tumor. $(\mathrm{H})$ The Ki-67 labeling index in tumor cells is very low $(<1 \%)$. 

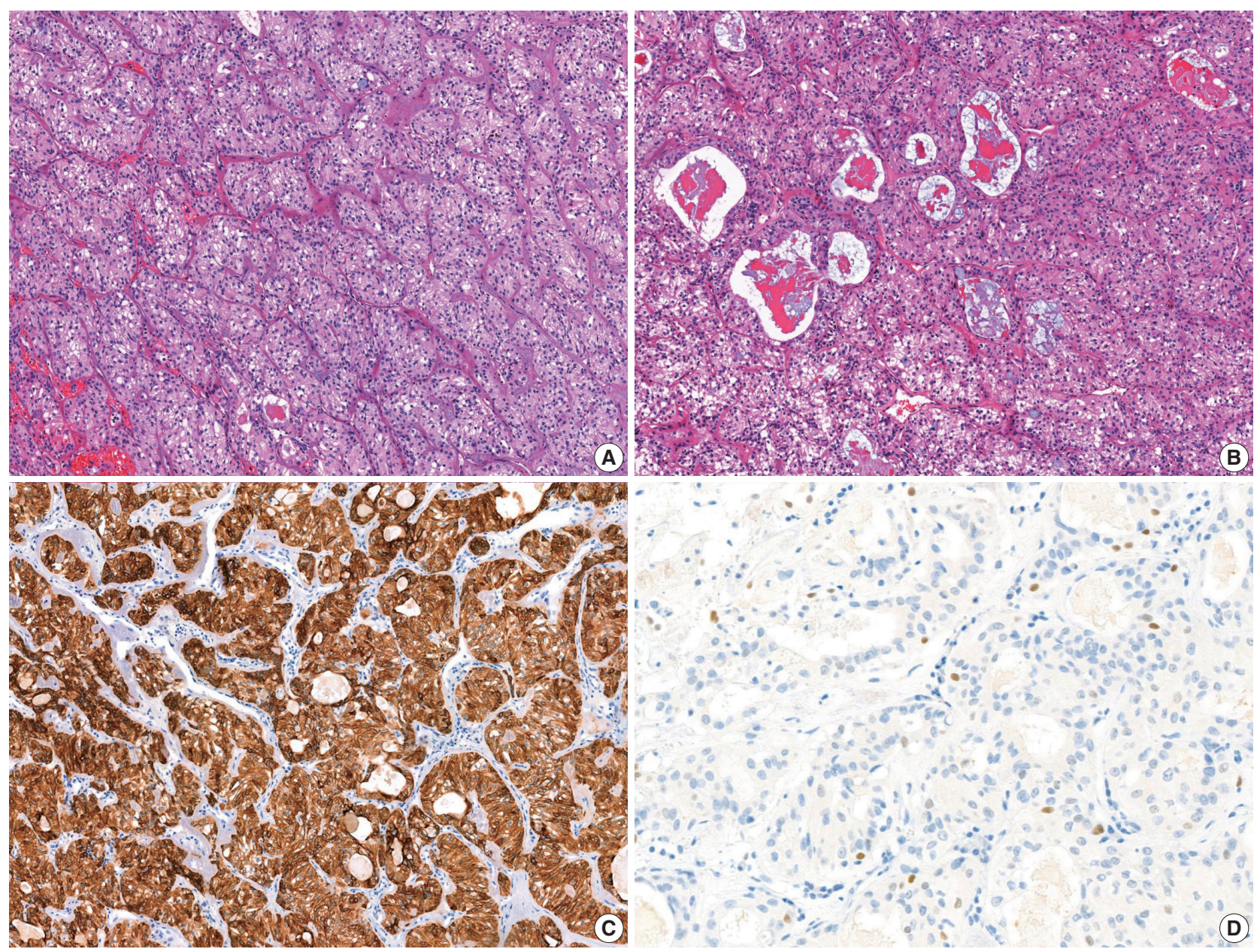

Fig. 2. Histopathological findings and immunostaining results for case 2. (A) Low-power image shows the tumor cells arranged in nests and cords which are surrounded by thick fibrous septa, forming trabecular growth pattern. The tumor cells possess lightly eosinophilic to clear cytoplasm. (B) Glandular components with intraluminal and intracytoplasmic mucin secretion are found in multiple areas. (C) Cytokeratin (AE1/AE3) staining reveals diffuse strong positive reactivity. (D) Focal p63 expressions are found in only a few tumor cells.

NPM1, NF1, MAP2K4, HSP90AA1, and GNAQ. Seventeen variants were nonsynonymous SNVs, and two were stop-gain $\mathrm{SNV}$ and non-frameshift deletion. Despite the high tumor purity $(>80 \%)$, all of the alterations had very low variant allele frequency $(<5.0 \%)$, and these variants were regarded as subclonal changes with genetic heterogeneity of tumor cells or possible sequencing errors.

\section{DISCUSSION}

Classification of salivary gland tumors is based mainly on histomorphology, lineage, and biological features [1]. Although no single antibody is specific for a tumor entity, the combination of immunohistochemical markers is helpful in the differential diagnosis of salivary gland neoplasms. With advances in the molecular diagnostics, specific genetic alterations in salivary gland tumors have been identified, and the classification is being further refined [3].

The two cases of salivary gland tumors reported here displayed unique histological changes that have not been described previously. The tumors displayed a corded and nested growth pattern with focal glandular differentiation and prominent vascularization. They had bland, round nuclei with inconspicuous nucleoli, and eosinophilic or clear cytoplasm. One of the two cases displayed pseudorosette-like arrangements around thickened blood vessels. Immunohistochemical staining for cytokeratin, SMA, S-100, c-KIT, p63, AR, and neuroendocrine markers revealed epithelial differentiation of the tumor cells, which were devoid of a myoepithelial component. Because this histological feature does not fit into any known type of salivary gland neoplasm, these tumors were initially diagnosed as adenocarcinoma, not otherwise specified.

Characteristic chromosomal rearrangements are found in many 

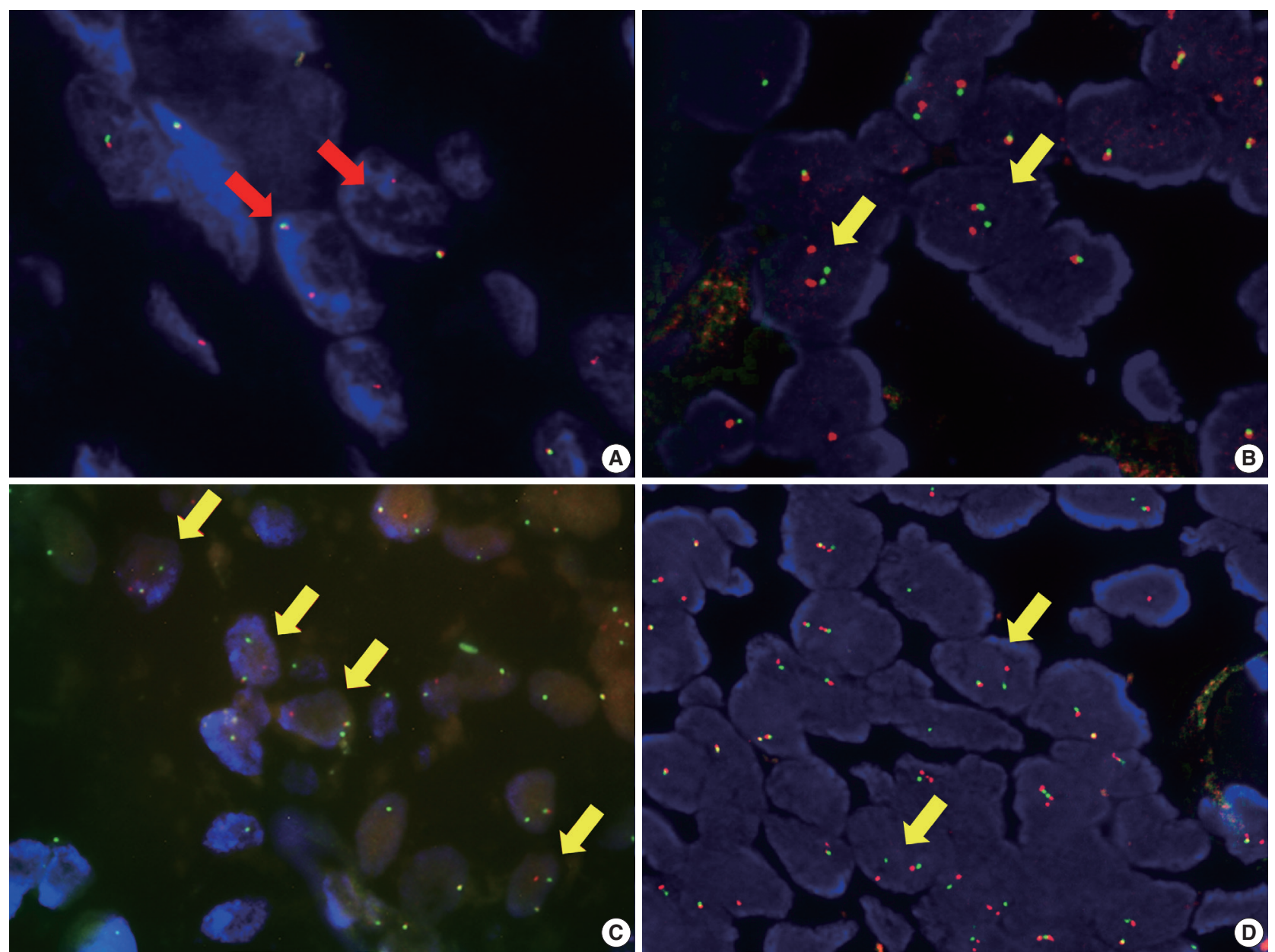

Fig. 3. Representative image of fluorescence in situ hybridization (FISH) analysis for MAML2 and EWSR1 from case 1 (A, B) and 2 (C, D). (A, B) In case 1, FISH analysis for MAML2 reveal one fused signal and a single red signal (red arrows), suggesting partial deletion or unbalanced translocation of MAML1 (A). (B) EWSR1 shows one fused signal and separate red and green signal (yellow arrows), indicating translocation of EWSR1. (C, D) Case 2 shows several cells with split of red and green signals (yellow arrows) for MAML2 (C) and EWSR1 (D), indicating concurrent MAML1 and EWSR1 rearrangement.

salivary gland neoplasms. For example, CRTC1-MAML2 fusion in mucoepidermoid carcinoma (MEC), ETV6-NTRK3 fusion in secretory carcinoma, $M Y B-N F I B$ fusion in adenoid cystic carcinoma, and EWSR1-ATF1 fusion in clear cell carcinoma (CCC) are well-known translocations and they are diagnostic for each tumor [4]. However, the presence of dual gene rearrangement in tumors of the salivary gland is extremely rare. Currently, there has been only one case report of intraosseous maxillary tumor harboring MAML2 and EWSR1 rearrangement [2]. The tumor consisted of sheets and nests of clear tumor cells with intervening hyalinized and desmoplastic septa. Epidermoid cells and mucinous cells were scattered throughout the tumor. FISH analysis showed rearrangement of both MAML2 and EWSR1 genes. Based on histomorphology and the cytogenetic findings, Hamza et al. [2] presumed this tumor as a hybrid of clear cell variant of MEC and clear cell odontogenic carcinoma. Similarly, in our case, positive results of MAML2 and EWSR1 analysis suggest the possibility of MEC or CCC. Interestingly, tumor cells showing one fused signal and a single red signal of $M A M L 2$ probe were frequently observed in one case, which could represent partial deletion or unbalanced translocation of the MAML2 gene. This unusual FISH pattern has also been previously reported in high-grade MEC [5].

MEC is the most common malignant salivary gland tumor. They are composed of varying proportions of squamoid, mucinproducing, and intermediate cells forming cystic and solid patterns. They are classified as low, intermediate, and high-grade, and the grading criteria include the proportion of mucous-cells, tumor invasiveness, anaplasia, mitotic rates, tumor necrosis, perineural or lymphovascular invasion, and the proportion of cystic component. Low-grade MECs are usually well circumscribed, 
mucous cell-rich, and have a prominent cystic component [6-8]. Most MECs are characterized by specific gene fusions involving $M A M L 2$, with a high tendency in low to intermediate grade tumors $[9,10]$. In our case, the tumor cells are mainly arranged in cords and nests exhibiting trabecular-like pattern, and partly form glands with intracytoplasmic and luminal mucin production. Although well-circumscribed tumor border and the presence of mucin-producing cells are reminiscent of low-grade MEC, epidermoid cells or intermediate cells which are helpful in diagnosis of MEC are not observed. Furthermore, variants of MECs known to date (oncocytic, Warthin-like, ciliated, and sclerosing) [10-13] did not cover the histological characteristics as in our cases.

CCC is a low-grade salivary gland carcinoma, frequently arising in the minor salivary glands, particularly in the base of the tongue. CCCs are composed of malignant cells with solid sheets, nests, cords, and trabecular growth pattern surrounded by hyalinizing stroma. Tumor cells may also show mucinous differentiation either focally or diffusely [14]. Diffuse and strong p63reactivity and EWSR1-ATF1 gene fusion is consistently identified in CCC [15]. In our patients, most tumor cells showed a corded and nested configuration with some glandular differentiation, and cytoplasmic clearing were partly observed. However, immunohistochemical staining revealed a lack of p63 expression in both cases, which does not match pathological characteristics of CCC.

In our cases, we used break apart probes to identify rearrangement of MAML2 and EWSR1, but unfortunately, we could not reveal the fusion partners. Nevertheless, based on positive FISH results of both genes, we suspect that these tumors possibly possess pathologic and molecular characteristics of both MEC and CCC. Identifying the specific fusion partner of MAML2 and EWSR1 would be helpful in refining this tumor entity.

The Ki-67 labeling index has been reported as a prognostic indicator and may be used for the differential diagnosis between malignant and benign tumors of the salivary gland $[16,17]$. Ki67 labeling index is usually $<5 \%$ in benign tumors and $>10 \%$ in malignant tumors. The tumors described here had a low Ki67 labeling index-both $<5 \%$. Based on the Ki-67 labeling index, this tumor could be defined as a benign neoplasm. However, histologic features, especially lymphovascular or capsular invasion, support that these tumors are malignant. The very low Ki-67 labeling index suggests that these tumors are low-grade malignancy. A larger number of cases with long-term follow-up is needed to determine the exact prognosis.

We identified 19 somatic variants (TOP1, PMS2, NPM1, NF1, MAP2K4, HSP9OAA1, and GNAQ) in the samples from patient 1 . These variants have not been previously reported for any other types of salivary gland tumors and may be associated with neoplastic transformation or progression. However, allele frequency of these variants was so low that it is doubtful that these genes have any diagnostic significance.

In conclusion, we have presented two cases of adenocarcinoma of minor salivary glands with concurrent MAML2 and EWSR1 alteration, which displayed unique histological changes that have not been previously described in other types of salivary gland neoplasms. Cords, nests, and trabecular-like arrangements of tumor cells are predominant, with foci of glandular components composed of mucous-cells. Well-circumscribed border, bland tumor nuclei, low Ki-67 labeling index, and non-aggressive clinical behavior suggest that these tumors are low-grade malignancies. Further investigations involving more cases sharing similar histological and molecular features are needed to define this unusual type of salivary gland tumor.

\section{Ethics Statement}

This study was approved by the Institutional Review Board of Samsung Medical Center with a waiver of informed consent (IRB No. 2020-02-146001) and was performed in accordance with the principles of the 1964 Helsinki Declaration.

\section{ORCID}

Sangjoon Choi https://orcid.org/0000-0003-2108-0575 Junhun Cho https://orcid.org/0000-0002-6089-9340 Seung Eun Lee https://orcid.org/0000-0002-7459-0061 Chung-Hwan Baek https://orcid.org/0000-0003-3602-4699 Yi-Kyung Kim https://orcid.org/0000-0002-9395-4879 Hyung-Jin Kim https://orcid.org/0000-0003-3576-3625 Young Hyeh Ko ～https://orcid.org/0000-0002-4383-0579

\section{Author Contributions}

Conceptualization: YHK. Data curation: SC, JC, SEL, CHB, YKK, HJK, YHK. Formal analysis: SC, JC, YHK. Investigation: SC, JC. Visualization: SC. Writing-original draft: SC, YHK. Writing_review \& editing: SC, YHK. Approval of final manuscript: all authors.

\section{Conflicts of Interest}

The authors declare that they have no potential conflicts of interest.

\section{Funding Statement}

No funding to declare.

\section{Acknowledgments}

We are grateful to Dr. Kyung-Ja Cho (Department of Pathology, Asan Medical Center) for providing MAML2 FISH analysis with valuable comments.

\section{References}

1. El-Naggar AK, Chan JK, Grandis JR, Takata T, Slootweg PJ. WHO classification of head and neck tumours. Lyon: IARC Press, 2017.

2. Hamza A, Yao CM, Lai SY, Bell D. Clear cell tumor of the maxilla 
with MAML2 and EWSR1 gene rearrangements: a true hybrid or a flourescence in situ hybridization fumble? AJSP Rev Rep 2020; 25: $12-5$.

3. Kato S, Elkin SK, Schwaederle M, et al. Genomic landscape of salivary gland tumors. Oncotarget 2015; 6: 25631-45.

4. Skalova A, Stenman G, Simpson RH, et al. The role of molecular testing in the differential diagnosis of salivary gland carcinomas. Am J Surg Pathol 2018; 42: e11-27.

5. Cipriani NA, Lusardi JJ, McElherne J, et al. Mucoepidermoid carcinoma: a comparison of histologic grading systems and relationship to MAML2 rearrangement and prognosis. Am J Surg Pathol 2019; 43: 885-97.

6. Auclair PL, Goode RK, Ellis GL. Mucoepidermoid carcinoma of intraoral salivary glands: evaluation and application of grading criteria in 143 cases. Cancer 1992; 69: 2021-30.

7. Hicks MJ, el-Naggar AK, Flaitz CM, Luna MA, Batsakis JG. Histocytologic grading of mucoepidermoid carcinoma of major salivary glands in prognosis and survival: a clinicopathologic and flow cytometric investigation. Head Neck 1995; 17: 89-95.

8. Goode RK, Auclair PL, Ellis GL. Mucoepidermoid carcinoma of the major salivary glands: clinical and histopathologic analysis of 234 cases with evaluation of grading criteria. Cancer 1998; 82: 1217-24.

9. Jee KJ, Persson M, Heikinheimo K, et al. Genomic profiles and CRTC1-MAML2 fusion distinguish different subtypes of mucoepidermoid carcinoma. Mod Pathol 2013; 26: 213-22.
10. Bishop JA, Cowan ML, Shum CH, Westra WH. MAML2 rearrangements in variant forms of mucoepidermoid carcinoma: ancillary diagnostic testing for the ciliated and Warthin-like variants. Am J Surg Pathol 2018; 42: 130-6.

11. Fujimaki M, Fukumura Y, Saito T, et al. Oncocytic mucoepidermoid carcinoma of the parotid gland with CRTC1-MAML2 fusion transcript: report of a case with review of literature. Hum Pathol 2011; 42: 2052-5.

12. Tasaki T, Matsuyama A, Tabata T, et al. Sclerosing mucoepidermoid carcinoma with eosinophilia of the salivary gland: case report and review of the literature. Pathol Int 2013; 63: 125-31.

13. Ishibashi K, Ito Y, Masaki A, et al. Warthin-like mucoepidermoid carcinoma: a combined study of fluorescence in situ hybridization and whole-slide imaging. Am J Surg Pathol 2015; 39: 1479-87.

14. Tanguay J, Weinreb I. What the EWSR1-ATF1 fusion has taught us about hyalinizing clear cell carcinoma. Head Neck Pathol 2013; 7: 28-34.

15. Weinreb I. Hyalinizing clear cell carcinoma of salivary gland: a review and update. Head Neck Pathol 2013; 7 Suppl 1: S20-9.

16. Larsen SR, Bjorndal K, Godballe C, Krogdahl A. Prognostic significance of Ki-67 in salivary gland carcinomas. J Oral Pathol Med 2012; 41: 598-602.

17. Luukkaa H, Klemi P, Leivo I, Vahlberg T, Grenman R. Prognostic significance of Ki-67 and p53 as tumor markers in salivary gland malignancies in Finland: an evaluation of 212 cases. Acta Oncol 2006; 45: 669-75. 